\title{
Time-Resolved Gas Temperatures in the Oscillating Turbulent Flow of a Pulse Combustor Tail Pipe ${ }^{*}$
}

\author{
JOHN E. DEC ${ }^{\dagger}$ \\ University of Michigan, Ann Arbor, Michigan
}

and

JAY O. KELLER

Combustion Research Facility, Sandia National Laboratories, Livermore, California

\begin{abstract}
The cyclic behavior of the gas temperature in the oscillating turbulent flow in a pulse combustor tail pipe was studied using two-line atomic fluorescence. In this flow, the oscillations result from an acoustic resonance, and have amplitudes of up to 5 times the mean velocity. Oscillation frequencies were varied from 67 to $101 \mathrm{~Hz}$. Spatially resolved temperature measurements were made to within $400 \mu \mathrm{m}$ of the wall, providing cycle-resolved profiles of the temperature and the random temperature fluctuations. The combustor-cycle phase relationships among the gas temperature, random-temperature-fluctuation intensity, velocity, and combustion chamber pressure, are compared. Velocity field effects dominated the cyclic behavior of the gas temperature, over the effects of isentropic compressive heating and the convection of hot pockets of gas from the combustion chamber. Cycle-resolved profiles show the gas temperature to be constant across the tail pipe, except for a boundary layer region, at all times during the cycle. Although cyclic temperature oscillations of more than $200 \mathrm{~K}$ were observed, the thermal boundary layer was well developed at all times during the cycle. The gas temperature was greater than the wall temperature at all cycle times, unlike the reversing velocity field, indicating that Reynolds analogy between momentum and thermal transport is not valid in this flow. Time-resolved wall heat flux was also measured and its fundamental oscillation is found to be in phase with that of the gas temperature.
\end{abstract}

\section{NOMENCLATURE}

$D \quad$ hydraulic diameter

$P \quad$ pressure

$P_{\text {rms }} \quad$ combustion chamber pressure, root mean square about the mean

* This work was performed at the Combustion Research Facility, Sandia National Laboratories and was supported by the U.S. Department of Energy, Energy Conversion and Utilization Technologies Program. John Dec was supported by the Gas Research Institute.

$\uparrow$ Currently at Sandia National Laboratories, Livermore, California

\begin{tabular}{|c|c|}
\hline & \\
\hline & seriod of a complete cycle \\
\hline$T$ & $\begin{array}{l}\text { gas temperature, instantaneous value, } \\
T(t)=\bar{T}+T_{\text {osc }}(t)+t^{\prime}(t)\end{array}$ \\
\hline$t^{\prime}$ & $\begin{array}{l}\text { random gas temperature fluctuations, in- } \\
\text { stantaneous value }\end{array}$ \\
\hline & gas temperature, time-averaged mean \\
\hline$\langle T\rangle$ & $\begin{array}{l}\text { gas temperature, ensemble-averaged } \\
\text { value, }\langle T\rangle(t)=\bar{T}+T_{\text {osc }}(t)\end{array}$ \\
\hline$\left\langle T^{\prime}\right\rangle$ & $\begin{array}{l}\text { random gas temperature fluctuations, } \\
\text { ensemble-averaged value }\left\langle T^{\prime}\right\rangle(t)= \\
\sqrt{\left\langle\left(t^{\prime}\right)^{2}\right\rangle}(t)\end{array}$ \\
\hline & $\begin{array}{l}\text { random gas temperature fluct } \\
\text { time-averaged mean of }\left\langle T^{\prime}\right\rangle(t)\end{array}$ \\
\hline
\end{tabular}

Published by Elsevier Science Publishing Co., Inc. 655 Avenue of the Americas, New York, NY 10010 
$T_{\text {center }}$ gas temperature at the tail pipe centerline

$T_{\text {osc }}$ gas temperature, ensemble-averaged oscillating component

$T_{\text {wall }} \quad$ wall temperature

$X \quad$ spatial variable along the axis of the tail pipe; $X=0$ is the contraction section-tail pipe interface.

$Y \quad$ vertical spatial variable normal to the tail pipe axis, $Y=0$ is at the wall

$Z$ horizontal spatial variable normal to the tail pipe axis, $Z=0$ is the tail pipe centerline.

\section{Greek Symbols}

$\delta_{T} \quad$ temperature defect boundary layer thickness

\section{INTRODUCTION}

Pulse combustion is an old technology that has recently received renewed attention in the search for high efficiency heating devices. Pulse combustor heating systems have many advantages over conventional burners, such as thermal efficiencies of $95 \%$ or more, low pollutant $\left(\mathrm{NO}_{x}\right.$ and $\mathrm{CO}$ ) emissions, self-aspiration, and high rates of heat transfer in the tail pipe. Although past research has provided a data base of overall pulse combustor performance, there is a lack of understanding of the fundamental controlling processes, and the coupling between these processes. As a result the design of pulse combustors has proceeded largely by trial and error, a process that is costly, rarely leads to optimal designs, and has limited the applications of these efficient burners. More complete discussions of pulse combustion applications and research needs may be found in Refs. 1-3.

One of the main advantages of pulse combustion is that the rates of heat transfer in the tail pipe are high, as much as 2.5 times greater than those of steady turbulent flow $[4,5]$. The large flow oscillations, which occur in the tail pipe of the pulse combustor as a result of the acoustic resonance of the device, are thought to be responsible for this enhancement, although the mechanism is not understood. It has been speculated [6] that the oscillating flow in the tail pipe alternately builds and breaks down the thermal boundary layer, thus preventing steady flow boundary layer development. The literature contains only time-averaged gas temperature measurements along the tail pipe centerline (for example, Ref. 4). There are no known studies of the temporal variations of the gas temperature, or the cyclic behavior of the thermal boundary layer in pulse combustor tail pipes or other strongly oscillating turbulent flows. Understanding the mechanisms of the heat transfer enhancement, and building accurate predictive models of the convection process, require detailed knowledge of the gas temperature field in the tail pipe.

Time-resolved gas temperature measurements will also provide insight into the magnitude and importance of cyclic temperature fluctuations (fluctuations of the gas temperature through the combustor cycle) in the pulse combustor tail pipe. There are several possible mechanisms for the generation of cyclic temperature fluctuations, for example: the convection of hot gas pockets produced in the combustion chamber by the cyclic combustion process, isentropic compressive heating, enthalpy flow due to the velocity oscillations, or cyclicly varying rates of convective heat transfer to the wall. The relative importance of these mechanisms and the magnitude of the cyclic temperature fluctuations is currently unknown. Understanding the causes and magnitudes of these cyclic temperature fluctuations will guide the development of computer models of pulse combustors, which are being developed to aid in the design process $[7,8]$. Accurate models of pulse combustors must properly predict the gas temperatures in the tail pipe, since the temperature field is coupled to all other processes through the speed of sound and the acoustic resonance of the combustor.

This article reports the first known timeresolved temperature data in a pulse combustor tail pipe or any strongly oscillating turbulent flow. A laser-based diagnostic recently developed by Dec and Keller [9], two-line atomic fluorescence (TLAF), was utilized to measure gas temperatures with a spatial and temporal resolution of $300 \mu \mathrm{m}$ and $500 \mu \mathrm{s}$, respectively. This resolution permitted measurements of the cyclic temperature fluctu- 


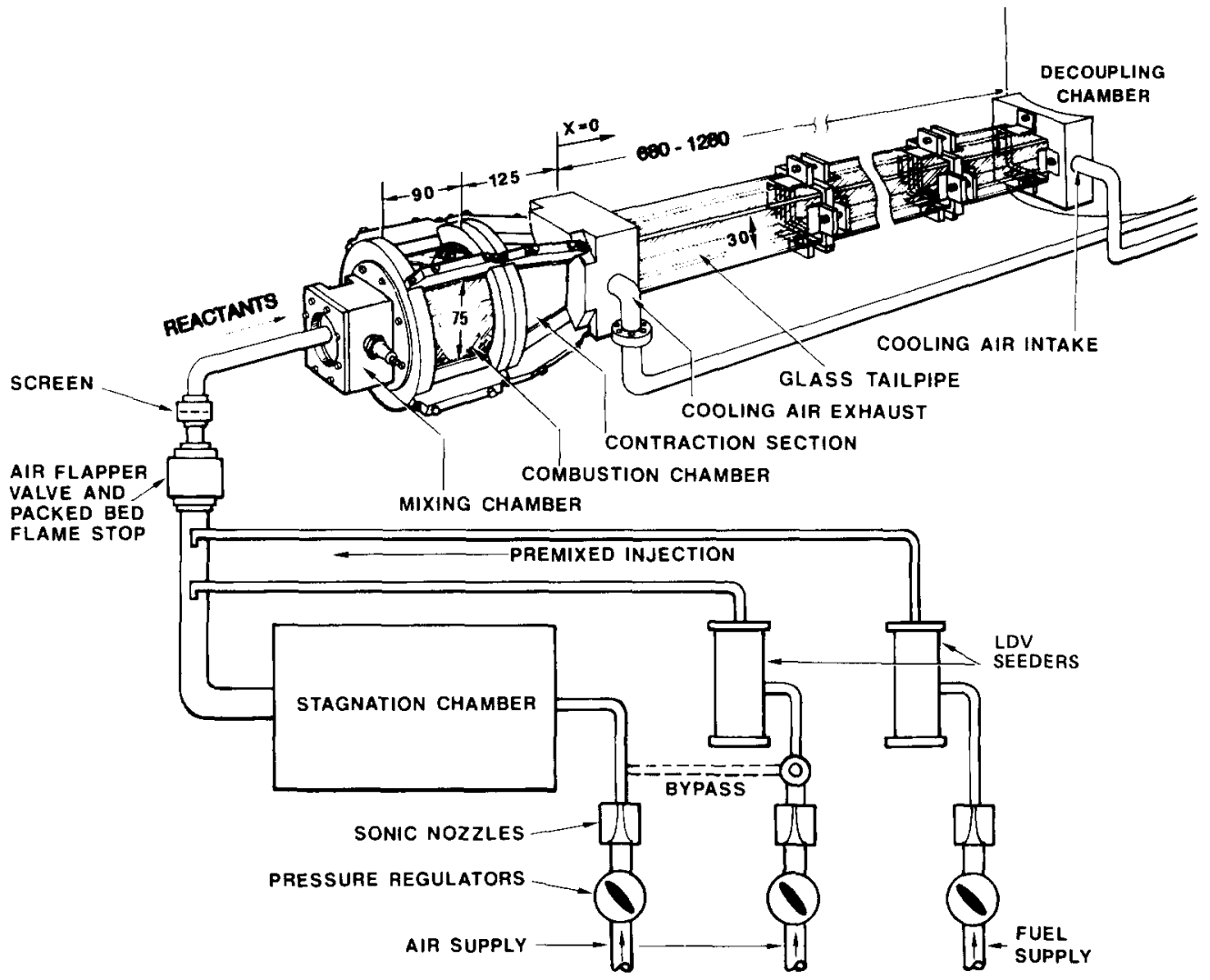

Fig. 1. Schematic of the pulse combustor facility, showing the variable length cooled tail pipe used in this study. All dimensions are in millimeters.

ations and cycle-resolved temperature profiles. In addition, the spatial and temporal resolution was sufficient to permit measurement of the random temperature fluctuations that are superimposed on the cyclic fluctuations in a flow that is turbulent as well as oscillating. These temperature data are also compared with cycle-resolved heat flux measurements. This study provides insight into the heat transfer mechanisms in pulse combustor tail pipes and a data base that will aid in the modeling of this flow.

\section{EXPERIMENTAL FACILITY AND DATA ACQUISITION}

\section{Experimental Facility}

The pulse combustor used for this study was a "Helmholtz" type with an external flapper valve. A schematic of the combustor is shown in Fig.
1. The combustor consists of an axisymmetric injection or "mixing" chamber, and square crosssection combustion chamber, contraction section, and tail pipe. Acoustical termination for the tail pipe was provided by a large-volume decoupling chamber. Reactants were premixed upstream of the flapper valve and injected on axis into the "mixing" chamber. Mean reactant mass flow rates were accurately metered with sonic nozzles. The mean reactant flow rate was held constant at 4.08 $\mathrm{g} / \mathrm{s}$ to yield a bulk mean Reynolds number in the tail pipe of about 3750 . The reactants consisted of pure methane and air at an equivalence ratio of 1.0 , and a variable fraction of nitrogen diluent. By varying the nitrogen diluent, varying the rate at which the reactants mixed with the products of the previous cycle, and varying the tail pipe length, the combustor could be operated at different frequencies and pulsation magnitudes, as discussed 
by Keller et al. [10, 11]. This technique permitted both strongly pulsing and steady flow conditions; however, the combustor would not operate stably across wide range of intermediate pulsation amplitudes. As a result, only a narrow range of pulsation amplitudes could be studied.

The tail pipe consisted of a coaxial square pyrex tube arrangement, as shown in the figure. In this arrangement, the inner tube, which was $30 \mathrm{~mm}$ square with a wall thickness of $4.75 \mathrm{~mm}$, formed the resonance tube for the pulse combustor and carried the combustion products. The outer tube was $51 \mathrm{~mm}$ square, which provided a $5.75 \mathrm{~mm}$ wide channel between the tubes on all four sides. Cooling air flowed through this square annular space between the inner and outer tubes, entering at the decoupler end of the tail pipe and exiting at the contraction section end. This tube arrangement therefore formed a counterflowing heat exchanger. The tail pipe was constructed in a modular fashion, allowing its length to be varied in $200-\mathrm{mm}$ increments from 680 to $1280 \mathrm{~mm}$, to obtain operating frequencies from 101 to $67 \mathrm{~Hz}$, respectively. This modular construction also permitted the insertion of two special test sections at 200-mm increments. One test section had optical quality walls for laser diagnostic access, and the other was equipped to measure time-resolved wall temperatures and heat fluxes. The inner tubes of these test sections were made of quartz rather than pyrex, which used in the rest of the tail pipe; the small difference in conductivity between these two materials had a negligible effect on the heat transfer.

The following coordinate system was used in this study. $X$ is along the axis of the tail pipe with the origin at the contraction section, tail pipe interface, as shown in Fig. $1 . Y$ is the vertical coordinate with its origin at the wall surface. $Z$ is the horizontal coordinate with its origin at the tail pipe centerline. All profiles presented are $Y$ profiles taken at $Z=0$. Measurements at the various $X$ positions, and $Y$ profiles, were obtained by moving the entire combustor, which was mounted on a three-dimensional translation stage. All times given for the cycle-resolved data have been normalized by the period of a complete cycle.

\section{Diagnostics}

In addition to the TLAF temperature measurement technique, laser Doppler velocimetry (LDV), pressure transducers, and thermocouples were used. The LDV system was used to measure centerline tail pipe velocities as described in Dec and Keller [12]. Pressure transducers were used to obtain the combustor frequency, and to measure the combustion chamber pressure. The root mean square about the mean of the combustion chamber pressure $\left(P_{\mathrm{rms}}\right)$ was used as a measure of the pulsation amplitude. Acoustic wave equations show, and Dec and Keller [12] experimentally verified, that $P_{\text {rms }}$ is directly related to the amplitude of the velocity oscillations in the tail pipe. In this article the term pulsation amplitude refers to both the velocity and pressure oscillations. The combustion chamber pressure was also used to establish a synchronizing trigger for the cycle-resolved measurements discussed below. The trigger defined time zero of the combustor cycle and was selected to occur at the downward-going zero-crossing of the oscillating component of the combustion chamber pressure.

Spatially and temporally resolved gas temperature measurements were made using TLAF. This technique utilizes two continuous-wave dye lasers tuned to pump two electronic transitions of atomic indium seed atoms. The resulting fluorescence emissions are at different wavelengths from the incident lasers, thereby eliminating elastic scattering from the windows, an important consideration in the narrow tail pipe. Temperature is determined from the ratio of the two fluorescence intensities using Boltzmann statistics. Calculating the temperature from the ratio of the fluorescence intensities eliminates the need to know the collisional quenching rate and the indium seed density. This is a convenient result since these values are difficult to quantify. A complete description of this diagnostic technique may be found in Dec and Keller [9]. In the current study, all data were taken at a rate of $2 \mathrm{kHz}$, providing a sufficient period of integration to minimize shot noise. A simple one-point calibration method was used in which the mean temperature $(\bar{T})$ obtained with the TLAF system 
was matched to that of a radiation corrected thermocouple [9]. (The maximum radiation correction was $2.5 \%$ of the gas temperature.) This calibration method eliminates the need to determine the efficiency of the collection optics and the photon to volts conversions of the individual photodetectors, values which are difficult to measure directly.

Thermocouples were used to obtain the cycleresolved heat flux. Wall surface temperatures were measured with a thin-film thermocouple, vacuum deposited on the exhaust gas side surface of the inner tube of the tail pipe. This thermocouple, made of pure platinum/pure rhodium, was $10 \mathrm{~nm}$ thick; hence, its effect on the heat flux and wall temperature was negligible. The time constant of this thermocouple was less than $100 \mu \mathrm{s}$, sufficiently fast to obtain cycle-resolved wall temperatures. A second thermocouple was embedded in the wall, at a known distance, directly below the thin film junction. It was not necessary to have a fast response thermocouple at this location since calculations showed that surface temperature oscillations, at the frequencies of interest, would be attenuated to $1 \%$ of their surface values in a distance much less than the $2 \mathrm{~mm}$ that separated the two thermocouples. The cycle-resolved heat flux was obtained from these two thermocouple measurements by solving the transient conduction equation in the wall.

\section{Terminology and Data Acquisition}

In an oscillating turbulent flow, the instantaneous values of the gas temperature $(T)$ is defined to be composed of three terms.

$T(t)=\bar{T}+T_{\mathrm{osc}}(t)+t^{\prime}(t)$,

where $T(t)$ is the instantaneous value, $\bar{T}$ is the long-time average mean, $T_{\text {osc }}(t)$ is the periodic or oscillating component caused by the acoustic resonance, and $t^{\prime}(t)$ is the instantaneous value of the random temperature fluctuations, which are superimposed on the periodic oscillation. An ensemble average of the temperature over many cycles yields,

$\langle T\rangle(t)=\bar{T}+T_{\mathrm{osc}}(t)$, where $\langle T\rangle(t)$, the ensemble-averaged temperature, is still a function of time, since it varies through the cycle. Since $\left\langle t^{\prime}\right\rangle(t)=0$, the ensembleaveraged intensity of the random or fluctuations is given the symbol $\left\langle T^{\prime}\right\rangle(t)$, which is defined as,

$$
\left\langle T^{\prime}\right\rangle(t)=\sqrt{\left\langle\left(t^{\prime}\right)^{2}\right\rangle}(t) .
$$

In the above discussion, the functional dependence on time has been noted for clarity for all appropriate terms. Since $\langle T\rangle,\left\langle T^{\prime}\right\rangle$, and $T_{\text {osc }}$ are always functions of time through the cycle, the functional dependence will not be noted in the remainder of this article.

The cycle-resolved measurements of the gas temperature were determined by ensemble averaging the measurements over a minimum of 400 combustor cycles. The time delay of each temperature realization from the time-zero trigger was recorded and the data were sorted into appropriate time bins, based on their time of arrival. A probability distribution function (pdf) was then calculated for each time bin. The mean of each time-bin pdf is then $\langle T\rangle . \bar{T}$ was obtained by averaging $\langle T\rangle$ over the cycle, and $T_{\text {osc }}$ was obtained by subtracting $\bar{T}$ from $\langle T\rangle$. Similarly, the standard deviation of each pdf provides $\left\langle T^{\prime}\right\rangle$. Cycleto-cycle variation will be included in this measurement of the random fluctuations; however it is considered negligible, since both the intensity and slope of the random temperature fluctuations near time zero, close to the synchronizing trigger, are in good agreement with those late in the cycle. (See Fig. 4). If there were large cycle-tocycle variation, a higher value of $\left\langle T^{\prime}\right\rangle$ would be expected at the end of the cycle. Time-averaged values of the random fluctuations $\overline{T^{\prime}}$ were obtained by averaging $\left\langle T^{\prime}\right\rangle$ over the cycle.

The accuracy of each TLAF realization is shot noise limited, and is a function of signal strength [9]. The signal intensity in the pulse combustor tail pipe varied systematically through the combustor cycle, being quite low at times, especially in the near-wall region, preventing measurements closer than $400 \mu \mathrm{m}$. To obtain the true value of $\left\langle T^{\prime}\right\rangle$, the measured value of $\left\langle T^{\prime}\right\rangle$ must be corrected for shot noise. The shot noise of each fluorescence signal follows Poisson statistics, being inversely 
proportional to the square root of the signal intensity. In this study, the signal intensities of the individual fluorescence emissions were recorded, as were the temperatures. The proportionality constants for converting the square roots of these intensities to shot noises were determined from measurements in a constant temperature flow. (This was necessary since the conversions between the number of photons collected and the signal intensity output by the photodection systems were not calibrated directly.) Calculation of the temperature errors resulting from the shot noise were made using the method discussed in Ref. 9. Assuming that shot noise and $\left\langle T^{\prime}\right\rangle$ are uncorrelated events, the total measured $\left\langle T^{\prime}\right\rangle$ is the square root of the sum of the squares of the shot noise and $\left\langle T^{\prime}\right\rangle$ [13]. This is a reasonable assumption in the pulse combustor tail pipe where shot noise is significant only when the indium seed concentration is low, an event which does not correlate with large values of $\left\langle T^{\prime}\right\rangle$. Corrections for shot noise ranged from near zero for most conditions to about $50 \%$ of the measured $\left\langle T^{\prime}\right\rangle$ at locations of very weak signal. All $\left\langle T^{\prime}\right\rangle$ and $\overline{T^{\prime}}$ values presented in this article have been corrected for shot noise.

\section{RESULTS AND DISCUSSION}

Most data to be presented were obtained at a typical combustor operating condition and axial location, which will be referred to as the base condition and base location. The base condition is a tail pipe length of $880 \mathrm{~mm}$, a frequency of $83 \mathrm{~Hz}$, a $P_{\text {rms }}$ of $7.4 \mathrm{kPa}$, and a mean flow Reynolds number (based on the hydraulic diameter) of 3750 . The base location is $X=540 \mathrm{~mm}$. Data were also taken at other operating conditions and locations and will be compared to those of the base condition and location.

\section{Cyclic Temperature Fluctuations}

A $\langle T\rangle$-time history at the tail-pipe centerline, for the base condition and location is presented in Fig. 2. For phase reference, the combustion chamber pressure and tail pipe gas velocity are plotted. Although these temperature and velocity data were taken at the centerline, they may be considered

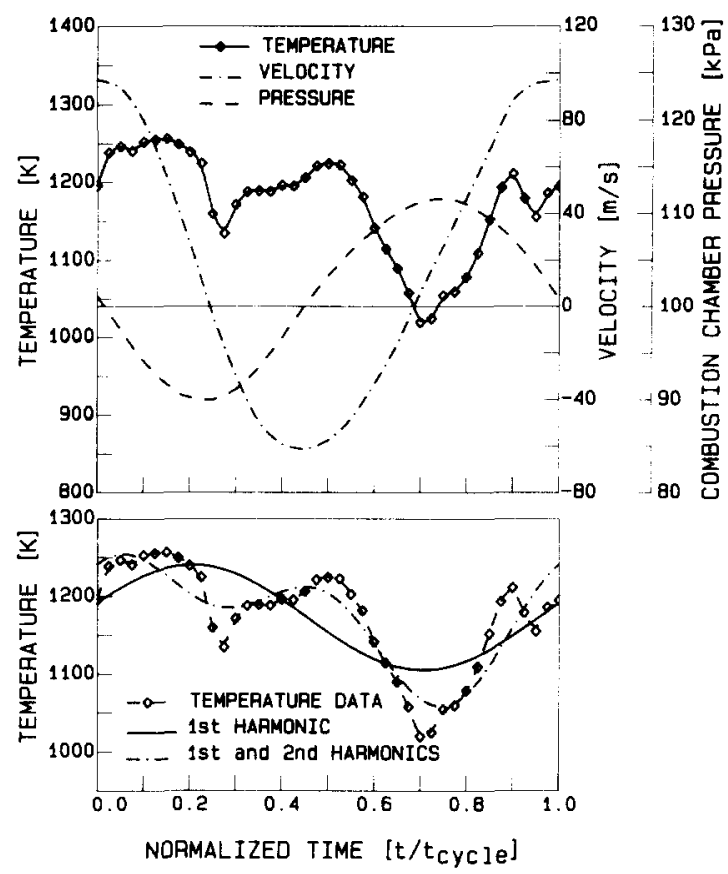

Fig. 2. A comparison of the cyclic behavior of the gas temperature and velocity at the tail pipe centerline, and the combustion chamber pressure, for the base operating condition and location. In the bottom plot are the temperature, its first harmonic, and sum of first and second harmonics from a Fourier series decomposition of the temperature fluctuations.

typical of the bulk values, since variation across the tail pipe is small except for the near wall region. This effect may be seen in the typical temperature profiles presented below, and in velocity profiles from this same combustor presented by Dec and Keller [12]. Temperature and velocity time histories for several different frequencies, axial locations, and pulsation magnitudes were also measured; their general appearance is similar to those presented in Fig. 2. This similarity indicates that the main features of the temperature-time history are caused by velocity oscillations, pressure effects, or heat transfer, which have the same phase relationship for all conditions.

It can be seen in the top plot of Fig. 2, that the combustion chamber pressure oscillation is roughly symmetric about time 0.5 and is sinusoidal. The velocity oscillation is strong, from +95 to $-60 \mathrm{~m} / \mathrm{s}$, with a mean of about $15 \mathrm{~m} / \mathrm{s}$. This velocity oscillation is 90 degrees out-of-phase with the combustion chamber pressure, as ex- 
pected from linear acoustic theory. The temperature oscillation is repeatable, but not very symmetric. Two features are present in this temperature field, although the detailed structure varies with operating condition. First, there is a fundamental oscillation at the combustor frequency, which is 90 degrees out-of-phase with the velocity and 180 degrees out-of-phase with the pressure. Second, superimposed on this oscillation are two dips (temporal locations of 0.25 and 0.7 ) in the temperature which slightly phase-lag the zero-velocity crossings.

The results of a Fourier series decomposition of the temperature, plotted in the bottom plot of Fig. 2, makes these features easier to visualize. In this figure, the solid line is the first harmonic or fundamental oscillation. The maximum in the first harmonic corresponds closely to the first zero-velocity crossing. At this time the gas at the probe volume has come from a maximum distance upstream (from the direction of the combustion chamber where it is hotter). The minimum in the first harmonic occurs near the second zero-velocity crossing when the gas in the probe volume has come from a maximum distance downstream (from the direction of the exit where it is cooler). Since this fundamental oscillation is almost 180 degrees out-of-phase with the pressure oscillation, it is attributed to the enthalpy flow effect described above. This effect dominates that of isentropic compressive heating, which produces in-phase temperature oscillations, and hence, dampens the measured temperature oscillations. The calculated isentropic compressive heating varies between $25 \%$ and $50 \%$ of the measured fundamental temperature oscillation for the various conditions tested. This result was found for all conditions tested.

The second feature of the temperature behavior, the dips at the zero-velocity crossings, occur twice each cycle, and hence, the magnitude of the second harmonic is related to the magnitude of these dips. The sum of the first and second harmonics can be seen to account for the major fluctuations in the temperature (bottom plot, Fig. 2). The cause of these temperature dips is not certain; however, a possible mechanism is a high rate of transport of energy to the wall, temporally correlated with the

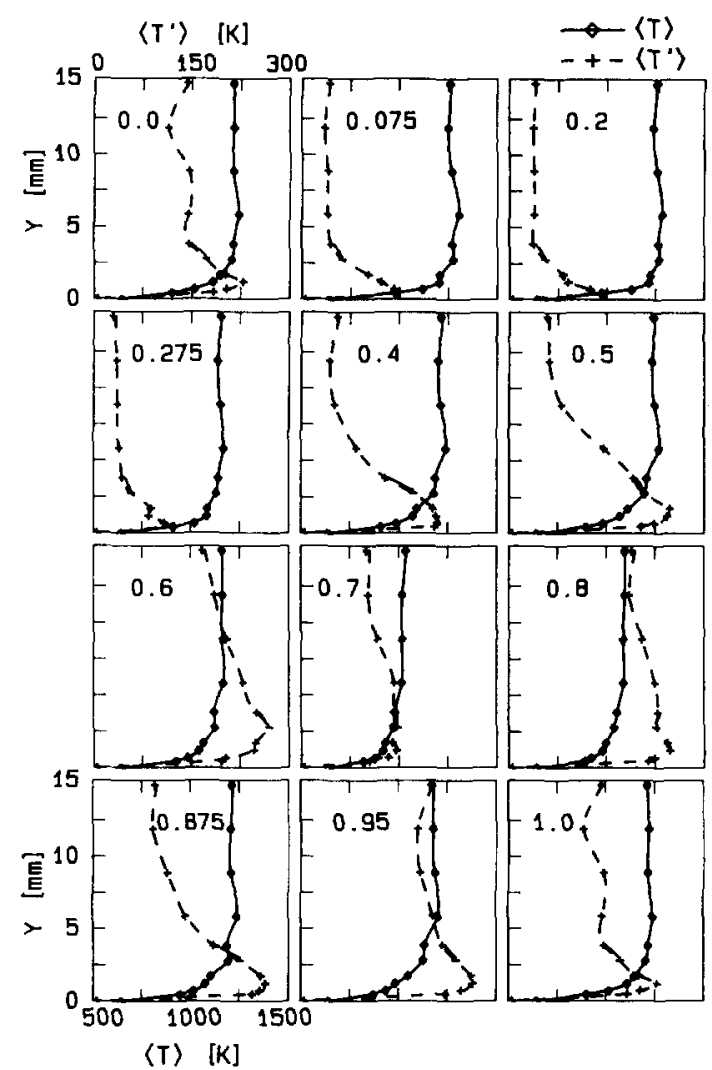

Fig. 3. Profiles of temperature $(\langle T\rangle)$ and random temperature fluctuations $\left(\left\langle T^{\prime}\right\rangle\right)$ at various times during the combustor cycle. Base operating condition and location.

zero-velocity crossing, a hypothesis corroborated by the heat flux data presented below.

\section{Cycle-Resolved Profiles}

Cycle-resolved spatial profiles of $\langle T\rangle$ and $\left\langle T^{\prime}\right\rangle$ are presented in Fig. 3 for the base condition and location. The cycle times selected for this figure correspond to times at which major changes occur in the time histories of either $\langle T\rangle$ or $\left\langle T^{\prime}\right\rangle$; see Fig. 5. Since TLAF temperature measurements were limited to $400 \mu \mathrm{m}$ from the wall, the wall temperature and $\left\langle T^{\prime}\right\rangle$ were set to the value measured with the thin film thermocouple and zero, respectively. Although the temperature difference between the centerline and the wall varies, the shape of the $\langle T\rangle$ profile does not change greatly during the cycle. The $\langle T\rangle$ profile can be divided into three regions, 
a conduction dominated sublayer next to the wall, approximately $1 \mathrm{~mm}$ thick, followed by a 1.5-3$\mathrm{mm}$ thick buffer layer and a core region where the temperature is virtually constant at the centerline value. In this article the conduction and buffer layers will collectively be referred to as the boundary layer. The greatest cyclic variation appears in the thickness of the buffer layer. This variation is most clearly seen in a comparison of the $\langle T\rangle$ profiles at times 0.2 and 0.5 . At time 0.2 the transition from the nearly linear conduction dominated sublayer to the constant temperature core is very abrupt with the temperature at $Y=2.5 \mathrm{~mm}$ being the same as the centerline value. In contrast, at time 0.5 the transition from conduction sublayer to core is gradual, and the temperature does not reach the core value until $Y=5 \mathrm{~mm}$.

These profiles show that, although there are variations in the nature of the thermal boundary layer, the thermal boundary layer is well developed throughout the cycle. Thus, the hypothesis that the increased heat transfer results from the thermal boundary layer being periodically broken down by the flow oscillations [6] is not valid. It can also be seen that $\langle T\rangle$ is greater than the wall value at all times during the cycle. In contrast, the velocity oscillates from positive to negative through the cycle (Fig. 2), while its wall value must remain zero. Hence, the time histories of the momentum and the thermal boundary layers are not similar, and it follows that the time histories of shear stress and heat fluxes are also dissimilar. Reynolds analogy, which is the basis of many heat transfer correlations, requires that the governing equations and boundary conditions of the momentum and energy equations be similar. Therefore, Reynolds analogy, or any heat transfer correlation based on this analogy is not valid for this flow.

Unlike $\langle T\rangle$, the $\left\langle T^{\prime}\right\rangle$ profiles, in Fig. 3, vary greatly over the cycle. At times 0.075 through $0.275,\left\langle T^{\prime}\right\rangle$ is constant at a low value to within about 3 or $4 \mathrm{~mm}$ of the wall. As the velocity approaches its maximum negative value, at times 0.4 and $0.5,\left\langle T^{\prime}\right\rangle$ in the near wall region increases and this wall effect penetrates approximately 10 $\mathrm{mm}$ into the flow, much farther than the thermal boundary layer. As the magnitude of the velocity decreases and goes through zero, at times 0.6 to $0.7,\left\langle T^{\prime}\right\rangle$ becomes more uniform across the tail pipe with the centerline value increasing to the near wall value. Then, as the velocity becomes more positive, time 0.875 , the profile is similar to times 0.4 and 0.5 when the magnitude is about the same. As the magnitude of the velocity increases further, at times 0.95 and 1.0 , there is an increase in $\left\langle T^{\prime}\right\rangle$ in the central core region. $\left\langle T^{\prime}\right\rangle$ then decreases rapidly, so that at time 0.075 the core region is constant at a much lower value, even though the velocity is still at a high value.

Although the $\left\langle T^{\prime}\right\rangle$ profiles in Fig. 3 vary through the cycle, there is a general trend for $\left\langle T^{\prime}\right\rangle$ to be a maximum in the boundary layer region, and decrease towards the centerline. Random temperature fluctuations are generated when gases from hotter and cooler regions are mixed by velocity fluctuations. In the boundary layer there are both large thermal and velocity gradients. Large velocity gradients are a mechanism for generating turbulent velocity fluctuations, which can then mix the different temperature gases across the boundary layer and give rise to the high $\left\langle T^{\prime}\right\rangle$ in the boundary layer.

Figure 4 presents $\langle T\rangle$ and $\left\langle T^{\prime}\right\rangle$ time histories at three representative transverse locations. $Y=15$ $\mathrm{mm}$, the centerline value, $Y=3 \mathrm{~mm}$ near the edge of the buffer layer, and $Y=0.8 \mathrm{~mm}$ in the conduction-dominated sublayer. The temperature behavior at $Y=3 \mathrm{~mm}$ has the same character as that of the centerline, but the amplitude of the oscillation is larger. In the conduction sublayer ( $Y=0.8 \mathrm{~mm}$ ) the temperature is cooler and variation during the cycle is relatively small.

In contrast to $\langle T\rangle,\left\langle T^{\prime}\right\rangle$ has a similar structure in the near wall $(Y=0.8 \mathrm{~mm})$ and edge of the thermal boundary layer ( $Y=3 \mathrm{~mm}$ ) regions, but is quite different at the centerline. At the edge of the boundary layer $(Y=3 \mathrm{~mm}),\left\langle T^{\prime}\right\rangle$ is characterized by two periods of increased intensity. The first, from time 0.25 to 0.7 , corresponds to the time when the velocity is negative. (See Fig. 2 for the velocity plot.) The second period of high $\left\langle T^{\prime}\right\rangle$, from time 0.7 to about 0.05 , roughly corresponds with the positive velocity period, which occurs from time 0.7 to 0.25 . However, $\left\langle T^{\prime}\right\rangle$ peaks at approximately time 0.9 , before the velocity max- 

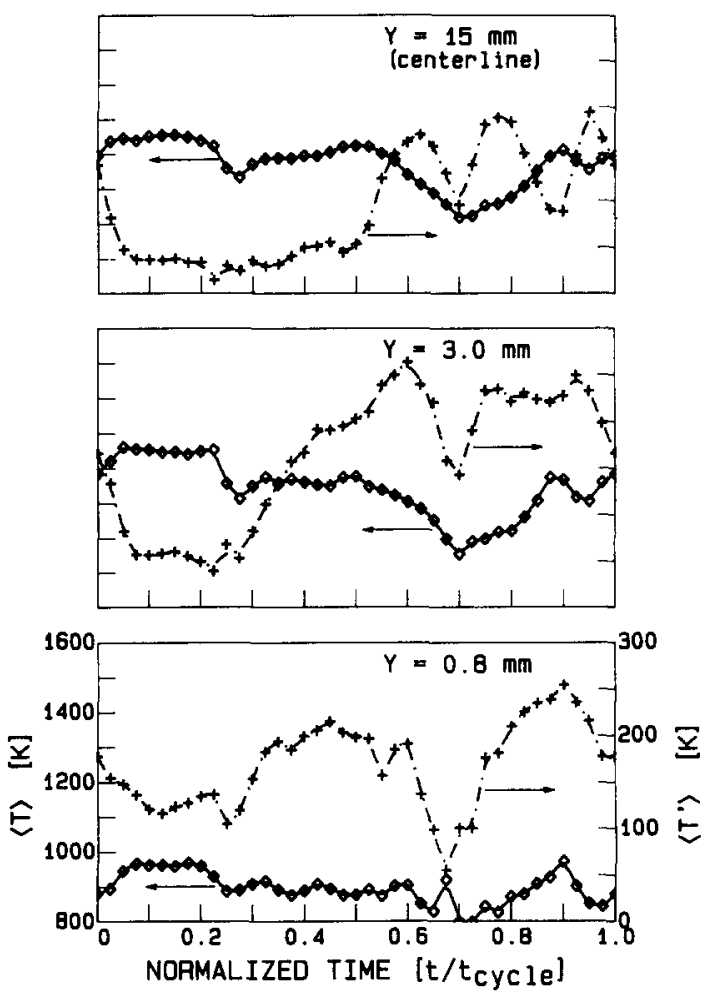

Fig. 4. Variations in $\langle T\rangle$ and $\left\langle T^{\prime}\right\rangle$ through and the cycle. Data are presented at three transverse locations, the centerline, the edge of the thermal boundary layer, and in the conduction sublayer. Base operating condition and location.

imum, and returns to a low level significantly before the zero-velocity crossing. The increased values of $\left\langle T^{\prime}\right\rangle$ in the wall region when the velocity magnitude is large might be caused by turbulent velocity fluctuations resulting from the increased shear present at this time of the cycle. The reason for the early decrease in $\left\langle T^{\prime}\right\rangle$ of the second period is not known. This effect, as well as all the previously described trends of the temperature and $\left\langle T^{\prime}\right\rangle$, were seen for all tail pipe locations, frequencies, and pulsation magnitudes studied. In contrast, the cyclic behavior of $\left\langle T^{\prime}\right\rangle$ in the centerline region varied with operating conditions and axial position, with the exception of an increase at about time 0.95 . This indicates that $\left\langle T^{\prime}\right\rangle$ in the core region does not arise directly from the velocity oscillations; perhaps it results from turbulent velocity fluctuations generated during the combustion process being convected down the tail pipe.

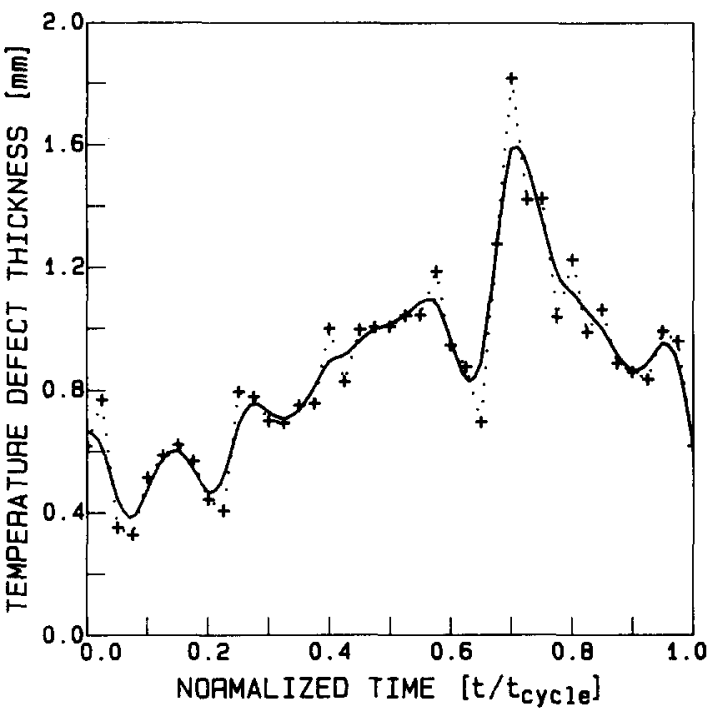

Fig. 5. Temperature defect thickness $\left(\delta_{T}\right)$ through the cycle for the base operating condition and location. The solid line is a cubic spline fit to the data, and the dotted line connects the data points.

To quantify cyclic variations of the thermal boundary layer observed in Fig. 3, a temperature defect thickness $\left(\delta_{T}\right)$ was calculated. $\delta_{T}$ is defined in the usual manner, as

$\delta_{T}=\int_{0}^{\text {center }}\left(1-\frac{T(y)-T_{\text {wall }}}{T_{\text {center }}-T_{\text {wall }}}\right) d y$,

where $T_{\text {wall }}$ and $T_{\text {center }}$ are the wall and the centerline temperatures, respectively. Figure 5 shows $\delta_{T}$ as a function of cycle time for the base condition and location. $\delta_{T}$ is small at time 0.2 , and large at time 0.5 , in agreement with the qualitative observations of the $\langle T\rangle$ boundary layers in Fig. 3. A comparison of Fig. 5 with the center and bottom plots of Fig. 4 shows that the cyclic fluctuations in $\delta_{T}$ are similar to those of $\left\langle T^{\prime}\right\rangle$ in the boundary layer. They are both small during the first part of the cycle, and begin increasing near the first zero-velocity crossing (time 0.25 ). After passing through local maxima, both $\delta_{T}$ and $\left\langle T^{\prime}\right\rangle$ have sharp local decreases near the second zero-velocity crossing (time 0.7 ). An explanation for the similar time-histories of $\delta_{T}$ and $\left\langle T^{\prime}\right\rangle$ is that increased convection occurs in the buffer layer with the same cyclic pattern. As this convection 

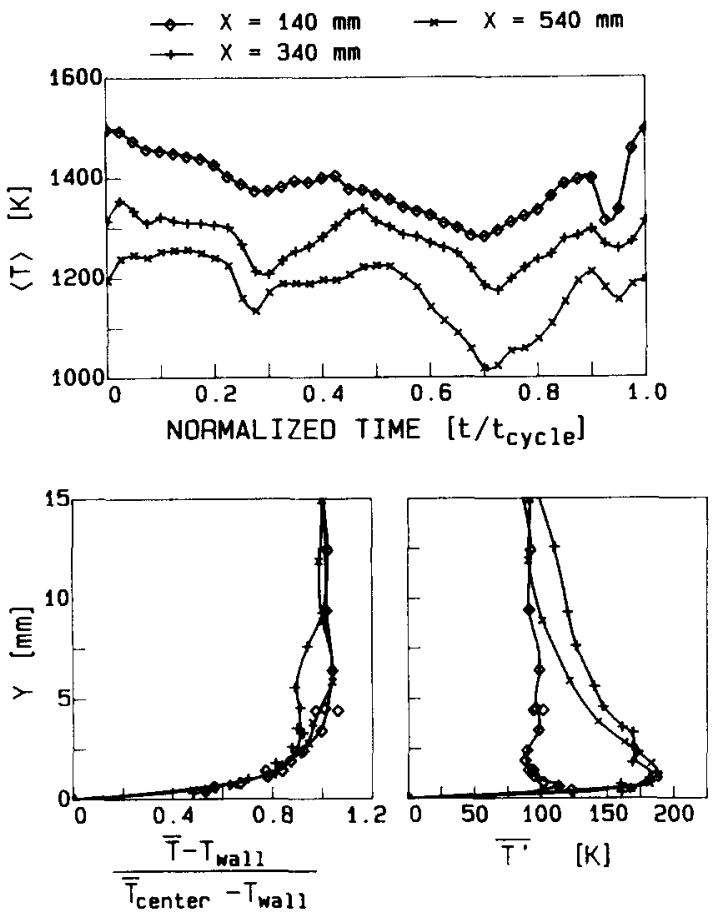

Fig. 6. Variations with axial location for the base operating condition. Shown are $\langle T\rangle$-time histories at the centerline (top), normalized time-averaged temperature profiles (lower left), and time-averaged intensity of random temperature fluctuations (lower right).

mixes the hotter and colder gases on either side of the buffer layer it causes an increase in $\left\langle T^{\prime}\right\rangle$, and smoothing of the transition from the conduction layer to the core, which increases $\delta_{T}$.

\section{Variation with Axial Position}

Variations in $\langle T\rangle, \bar{T}$, and $\overline{T^{\prime}}$ with axial position are shown in Fig. 6, for the base condition. Data are presented for the 140-, 340-, and 540-mm $X$ positions of this $880-\mathrm{mm}$ tail pipe; TLAF measurements could not be obtained at $X=740$ because the fluorescence signal was too weak. The top plot shows $\langle T\rangle$-time histories at the tail pipe centerline. Although there is some variation with axial position, the general pattern of the cyclic temperature fluctuations is the same. Normalized, time-averaged profiles of the difference between the gas and the wall temperature, presented in the lower left plot, exhibit remarkably little change with axial position, especially in the region near the wall. This is in agreement with the heat transfer study of Dec and Keller [5] in this flow, which showed the time-averaged Nusselt number to be virtually identical at these three axial locations.

Despite the similarity of the time-averaged temperature profiles the $\overline{T^{\prime}}$ profiles show some development with axial position. At $X=140 \mathrm{~mm}, \overline{T^{\prime}}$ is constant across the core and buffer layer, while farther from the entrance, $X=340$ and $540 \mathrm{~mm}$, $\overline{T^{\prime}}$ varies across the entire width of the tail pipe. Although the cycle-resolved $\left\langle T^{\prime}\right\rangle$ profiles (Fig. 3) vary considerably, the $\overline{T^{\prime}}$ profile is smooth. The $\overline{T^{\prime}}$ profile shape is similar to a typical streamwise velocity turbulence profile in steady flow. $\overline{T^{\prime}}$ rises rapidly through the conduction sublayer to reach a maximum at about the center of the $\bar{T}$ boundary layer, $1.5 \mathrm{~mm}$. $\overline{T^{\prime}}$ then gradually decays towards the centerline, where its magnitude is only about half the maximum value. These results are similar to the cycle-resolved data in that the wall effect on $\overline{T^{\prime}}$ penetrates in approximately $10 \mathrm{~mm}$ as opposed to $3 \mathrm{~mm}$ for the mean temperature.

\section{Variation with Frequency}

Figure 7 shows $\langle T\rangle$-time histories at the centerline for various frequencies. Pulsation amplitudes $\left(P_{\text {rms }}\right)$ were similar for all frequencies, and all data were taken at the base location ( $X=540$ $\mathrm{mm}$ ) except that of $101 \mathrm{~Hz}$, which was at $X=340$ mm due to weak TLAF signals at $X=540 \mathrm{~mm}$ in the short $680-\mathrm{mm}$ tail pipe used for the $101-\mathrm{Hz}$ data. The features of these curves are similar for all frequencies however, the magnitude of the fluctuations, particularly the dips at the zero-velocity crossings (cycle times of about 0.25 and 0.7 ), are larger at the higher frequencies than at the lower frequencies.

A possible source of temporal gas-temperature fluctuations in a pulse combustor tail pipe is the convection of hot gas pockets produced by the periodic combustion process [10]. If the streamwise mixing in the combustion chamber and contraction section was insufficient, the hot gases produced each combustor cycle could retain their identity. Since the average rate of travel of these hot pockets would be at the mean velocity, they would create temperature fluctuations whose phase relationship 


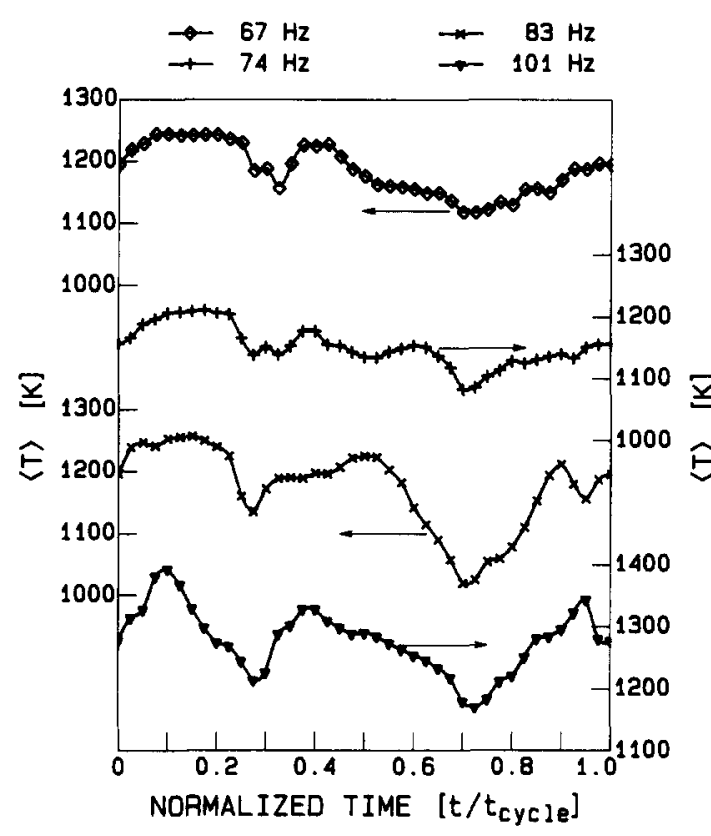

Fig. 7. Variation of $\langle T\rangle$ with time at the centerline for various combustor frequencies. All data are at the base location ( $X=$ $540 \mathrm{~mm}$ ), except that for $101 \mathrm{~Hz}$ which are at $X=340 \mathrm{~mm}$. The pulsation amplitude is similar for all cases.

varied with axial location and operating condition. Examination of the temperature-time histories in Figs. 6 and 7 shows that the basic features of the cyclic temperature fluctuations are the same for all axial locations and frequencies tested. Similar results were found when the pulsation amplitude was varied. This indicates that the convection of hot gas pockets down the tail pipe is not a dominant cause of gas-temperature fluctuations. Rather the cyclic fluctuations are dominated by the velocity, pressure, and heat transfer effects previously discussed.

Time-averaged profiles for the same frequencies and locations as Fig. 7 are presented in Fig. 8. Shown are cubic spline fits to the data points. The upper left plot shows the appropriately normalized time-averaged temperature profiles. Although the profiles have some random variation across the core, a trend may be seen in the boundary layer. The profiles for the $83-$ and $101-\mathrm{Hz}$ cases have steeper gradients at the wall than do those from 67 and $74 \mathrm{~Hz}$. This is in agreement with a Nusselt number increase with frequency observed in this flow [5]. There is little variation in the magnitude
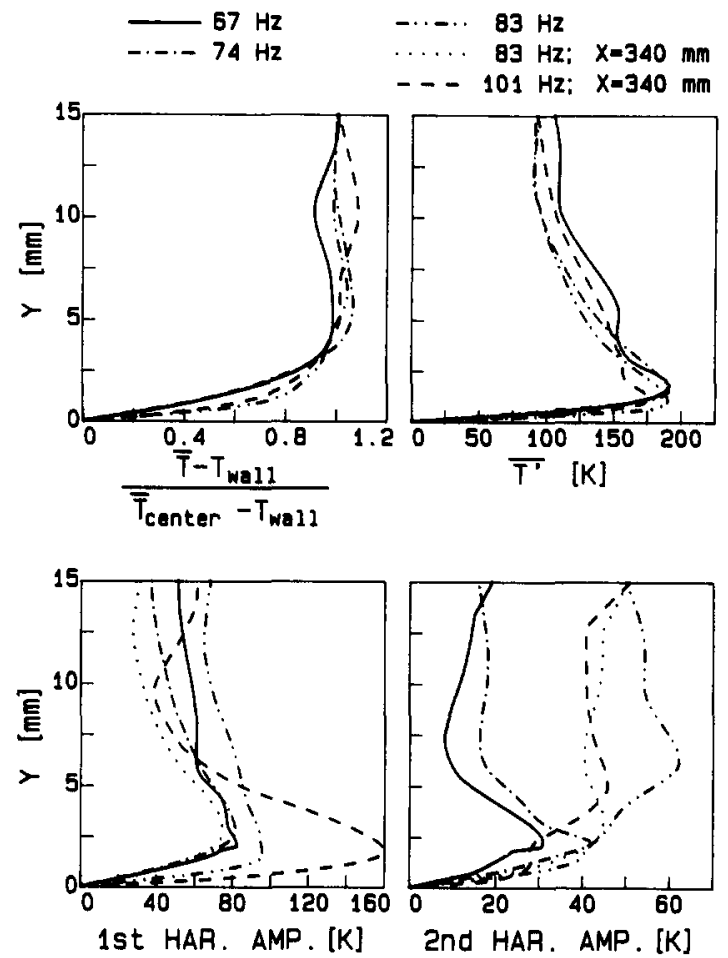

Fig. 8. Variations with frequency. Shown are normalized timeaveraged temperature profiles (upper left), time-averaged intensity of random temperature fluctuations (upper right), amplitude of the first harmonic (lower left), and amplitude of the second harmonic (lower right). All data are at the base location ( $X=540 \mathrm{~mm}$ ), except as noted. The pulsation amplitude is similar for all cases.

or shape of the $\bar{T}^{\prime}$ profiles with frequency (upper right plot).

The two lower plots in Fig. 8 show profiles of the amplitudes of the first and second harmonics of the $\langle T\rangle$-time histories. With the exception of the $101-\mathrm{Hz}$ case, the first harmonic profiles have a similar shape. The magnitude of the first harmonic increases rapidly near the wall to reach a maximum near the edge of the boundary layer ( $Y=2$ to $3 \mathrm{~mm}$ ), then decreases towards the centerline. Although the maximum magnitude of the first harmonic is about $80 \mathrm{~K}$ for all frequencies, they vary by nearly a factor of 2 in the core region, and have no clear trend with frequency. In contrast, the second harmonic amplitude profiles have different shapes, but show a trend of increasing with frequency. At the lower frequencies (67 and $74 \mathrm{~Hz}$ ) the second harmonics increase through 
a maximum at the edge of the boundary layer, then decrease sharply to a lower value across most of the core. For the higher frequencies ( 83 to 101 $\mathrm{Hz}$ ), the second harmonics increase through the boundary layer to their core values.

For $Y>2.5 \mathrm{~mm}$ the amplitude of the second harmonic increases with frequency, being lowest at $67 \mathrm{~Hz}$, higher at $74 \mathrm{~Hz}$, and more than a factor of 2 greater at $83 \mathrm{~Hz}$ for the $X=540 \mathrm{~mm}$ location. The $101-\mathrm{Hz}$ data that were taken at $X=340$ $\mathrm{mm}$ fall below the $83-\mathrm{Hz}$ data for $X=540 \mathrm{~mm}$; however, they have the same magnitude as the 83$\mathrm{Hz}$ data at $X=340 \mathrm{~mm}$ (also shown). The increasing magnitude of the second harmonic with frequency provides a quantitative measure of the qualitative observation made in the discussion of Fig. 7 above, that the magnitudes of the temperature dips at the zero-velocity crossings increase with frequency. This trend is the same as that of the convective heat transfer in the tail pipe, which the authors previously found [5] to increase with frequency. Further evidence that these temperature dips are related to the heat flux is shown in the next subsection.

\section{Comparison with Heat Flux}

A comparison of the gas temperature (measured by TLAF) and the wall heat flux (measured by a thin film thermocouple) is shown in Fig. 9. In this plot the centerline gas temperature, the wall heat flux and their first harmonics are shown. Since the temperature difference between the gas and the wall drives the heat transfer, and the wall temperature oscillates less than 1 degree, the heat flux would be expected to oscillate with the gas temperature, if the rate of convection were constant. It can be seen that the heat flux has a fundamental oscillation that is almost in phase with the fundamental oscillation of the temperature. There is however, a significant difference in the two curves. As noted in Fig. 2, at the times when the velocity crosses zero, there are distinct drops in the temperature. In Fig. 9 it can be seen that there are corresponding increases in the wall heat flux. Although the magnitude of this effect varied, it was noticed at all frequencies, pulsation amplitudes and axial positions studied. High rates of convec-

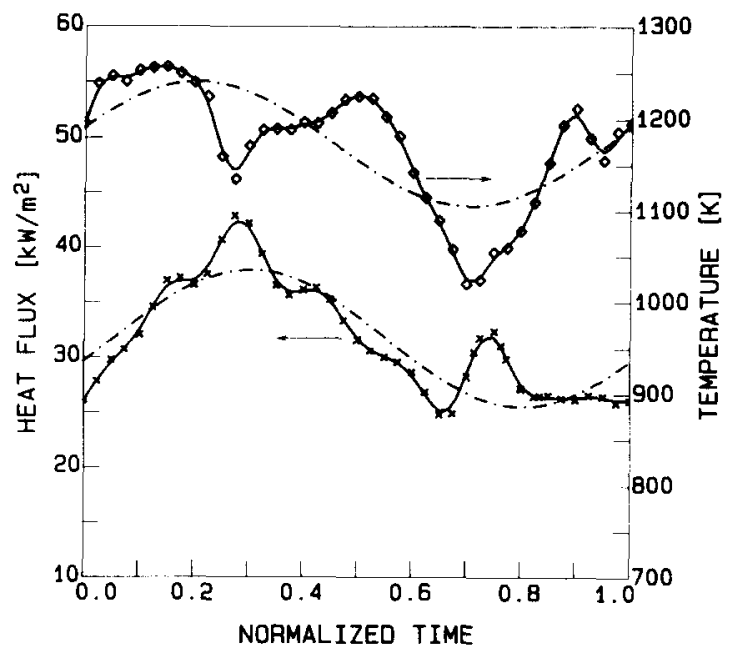

Fig. 9. A comparison of gas temperature oscillations at the tail pipe centerline with wall heat flux oscillations at the same tail pipe location. The first harmonic of both phenomena are also shown.

tive transport of thermal energy from the gas to the wall could explain this effect, but the fluid mechanics responsible are not well understood. This increase in transport is strong, since the temperature difference between the core gas and the wall drops as the heat flux increases.

\section{SUMMARY AND CONCLUSIONS}

A study of the time-resolved gas temperature in the tail pipe of pulse combustor was performed using two-line atomic fluorescence. This study showed that the tail pipe gas temperature oscillates with a characteristic pattern that may be described by the first two harmonics of the cycle-resolved data. The first harmonic results from the corresponding enthalpy flow as the gas at the probe volume comes alternately from the hot combustion chamber end and then from the cooler exit end of the tail pipe. The second harmonic is shown to describe two distinct dips in the temperature that occur at the times of flow reversal. Similar temperature fluctuations were noted at all frequencies, pulsation amplitudes, and axial locations, indicating that they were caused by the effects of velocity, which are also similar at all operating conditions and locations. These effects were dominant over insentropic compressive heating and the convec- 
tion of hot pockets of gas from the combustion chamber.

Cycle-resolved profiles (ensemble-averages of many combustor cycles) showed the gas temperature to be nearly constant across the tail pipe at all times during the cycle, except for a boundary layer region. Random temperature fluctuations superimposed on the cyclic oscillations were found to have a consistent cyclic pattern in the wall region but not in the central region of the tail pipe. This effect penetrated into the flow as much as 3 times farther than the thermal boundary layer. The thermal boundary layer was seen to be well developed throughout the cycle. Unlike the velocity, which reverses, the gas temperature is greater than its wall value at all times during the cycle. Therefore, any heat transfer correlation based on Reynolds analogy between momentum and thermal transport is not valid in this flow.

Normalized time-averaged temperature profiles were found to be similar for all axial locations measured, and showed a trend of increased temperature gradient at the wall with increased frequency. These findings are in agreement with a previous heat transfer study in this flow [5], which showed the Nusselt number to be similar for different axial positions, but to increase with frequency. No trends with frequency were found in the intensity of the random temperature fluctuations or in the amplitude of the first harmonic of the cyclic temperature oscillations. The amplitude of the second harmonic of the cyclic temperature oscillations was found to increase with frequency, suggesting that the fluid dynamics responsible for the temperature dips, which occur at times of flow reversal, may be linked to the heat transfer enhancement observed in some oscillating flows. Finally, the fundamental oscillation in the wall heat flux was found to correspond to the fundamental oscillation in the gas temperature. This indicates that cyclic variations in the Nusselt number are small except at times of flow reversal when the gas temperature drops and the heat flux increases.

\section{REFERENCES}

1. Putnam, A. A., Belles, F. E., and Kentfield, J. A. C., Prog. Ener. Combust. Sci. 12:43-79 (1986).

2. Zinn, B. T., ASME Paper 84-WA/NCA-19, ASME Winter Annual Meeting, New Orleans, LA, December, 1984.

3. Anon., Proceedings Vol. I: Symposium on Pulse Com. bustion Applications, GRI-82/0009.2, Atlanta, GA, March 1982.

4. Hanby, V. I., ASME J. Engr. Power 91:48-52 (1969).

5. Dec, J. E., and Keller, J. O., Combust. Flame 77:359-374 (1989).

6. Lalwani, P. C., Das, K. L., Bhattacharyya, T. K., and Bhaduri, D., Mech. Eng. Bull. 10:7-10 (Mar/June 1979).

7. Barr, P. K., Dwyer, H. A., and Bramlette, T. T., Combust. Sci. Technol. (in press).

8. Tsujimoto, Y., and Machii, N., Twenty-First Symposium (International) on Combustion, The Combustion Institute, Pittsburgh, 1986.

9. Dec, J. E., and Keller, J. O., Twenty-First Symposium (International) on Combustion, The Combustion Institute, Pittsburgh, 1986.

10. Keller, J. O., Dec, J. E., Westbrook, C. K., and Bramlette, T. T., Combust. Flame 75:33 (1989).

11. Keller, J. O., Westbrook, C. K., Bramlette, T. T., and Dec, J. E., Combust. Flame 79:151-161 (1990).

12. Dec, J. E. and Keller, J. O., Proceedings of the International Gas Research Conference, Toronto, Canada, Sept. 8-11, 1986 (also Sandia National Laboratories Report, SAND86-8757).

13. Kreyszig, E., Advanced Mathematics for Engineers and Scientists, Wiley, New York, 1972.

Received 4 January 1989; revised 7 August 1989 